\title{
Unraveling the Nature of Extraframework Catalytic Ensembles in Zeolites: Flexibility and Dynamics of the Copper-Oxo Trimers in Mordenite
}

\author{
Elena V. Khramenkova, Michael G. Medvedev, Guanna Li, and Evgeny A. Pidko*
}

Cite This: J. Phys. Chem. Lett. 2021, 12, 10906-10913

Read Online

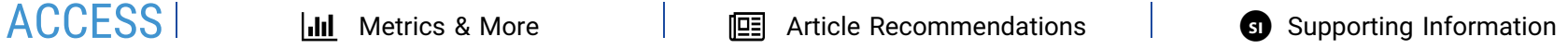

ABSTRACT: Extraframework cations define the chemical versatility of zeolite catalysts. Addressing their structural complexity and dynamic behavior represents one of the main fundamental challenges in the field. Herein, we present a computational approach for the identification and analysis of the accessible pool of intrazeolite extraframework complexes with a $\mathrm{Cu} / \mathrm{MOR}$ catalyst as an industrially important model system. We employ ab initio molecular dynamics for capturing the ensemble of reactive isomers with the $\left[\mathrm{Cu}_{3} \mathrm{O}_{3}\right]^{2+}$ stoichiometry confined in the mordenite channels. The high structural diversity of the generated isomers was ensured by concentrating the kinetic energy along the low-curvature directions of the potential energy surface (PES). Geometrically distinct $\left[\mathrm{Cu}_{3} \mathrm{O}_{3}\right]^{2+}$ complexes were identified via a series of clustering procedures ensuring that one structure of each local minima is retained. The proposed procedure has resulted in a set of previously unknown peroxo-complexes, which are $>50 \mathrm{~kJ} / \mathrm{mol}$ more stable than the recently hypothesized chair-shaped structure. Our analysis demonstrates that the most stable peroxo-containing clusters can be formed under operando conditions from molecular oxygen and the $\mathrm{Cu}_{3} \mathrm{O}$ unit, similar to that in methane monooxygenase (MMO) enzymes.

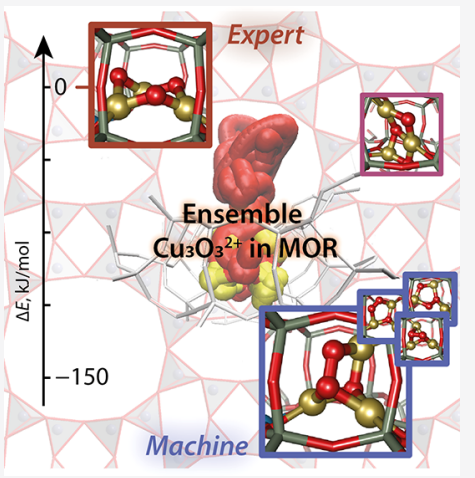

$\mathrm{T}$ he development of advanced predictive models in heterogeneous catalysis requires a deep insight into the molecular-level structure of the active sites, the mechanism of their action, and evolution under operating conditions. There is growing evidence of the critical role of dynamic phenomena for catalytic reactivity. When exposed to the reaction conditions, the reactive ensembles may evolve and reconstruct, therefore altering their chemical reactivity and catalytic behavior. ${ }^{1}$ Understanding the dynamics of the active sites induced by the environmental variables is key to the development of a detailed molecular picture of the catalytic phenomena and thus forms the basis for the rational design of new and improved catalyst systems. ${ }^{2}$

The view of the catalytic active site as a dynamic system conflicts with the conventional theoretical approaches. It is commonly assumed that the configurations corresponding to the global minima of the active site-the intrinsically most stable dominant configuration-are maintained during the reaction. This assumption is acceptable only when the catalytic site is truly rigid. ${ }^{3}$ However, a wider range of catalytic structures can emerge during the reaction in response to the exposure to the reactive environment. ${ }^{4,5}$ The latter includes such parameters as an elevated temperature that induces the thermal crossing of the energy barriers, the concentration of reactants and products, electrochemical potential, pressure, solvents, etc. Therefore, active site reorganization and its dynamics have to be accounted for when building an atomistic description of the catalytic system.

The dynamics in catalytic sites was spotted by Zhang et al., who extended the description of the catalytic models by representing a dynamic evolution of the ensemble of structures occurring under realistic conditions. ${ }^{6}$ In this sense, the isomerization of the catalyst introduces new reaction pathways with different mechanisms and rates. Catalyst activity, stability, and reactivity can be controlled by the presence of the less populated metastable states whose catalytic role is abominably underestimated.

The growing understanding of the role of metastable states encourages studying the properties of a catalytic system as ensemble averages, with contributions of all the coexisting configurations. Such a broader picture of the catalytic active sites is crucial to construct a comprehensive mechanistic representation beyond the field of heterogeneous catalysis as demonstrated recently for uncatalyzed reactions ${ }^{7,8}$ and homogeneous catalysis. ${ }^{9,10}$ A representative example of dynamic heterogeneous catalysis is $\mathrm{CO}$ oxidation by $\mathrm{MnO}_{x}$ -

Received: October 6, 2021

Accepted: October 27, 2021

Published: November 3, 2021

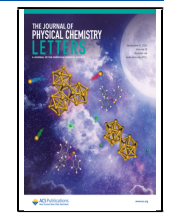


Scheme 1. Schematic Overview of the Proposed Computational Workflow for Automatic Structural Exploration of Extraframework Cations in the Zeolite

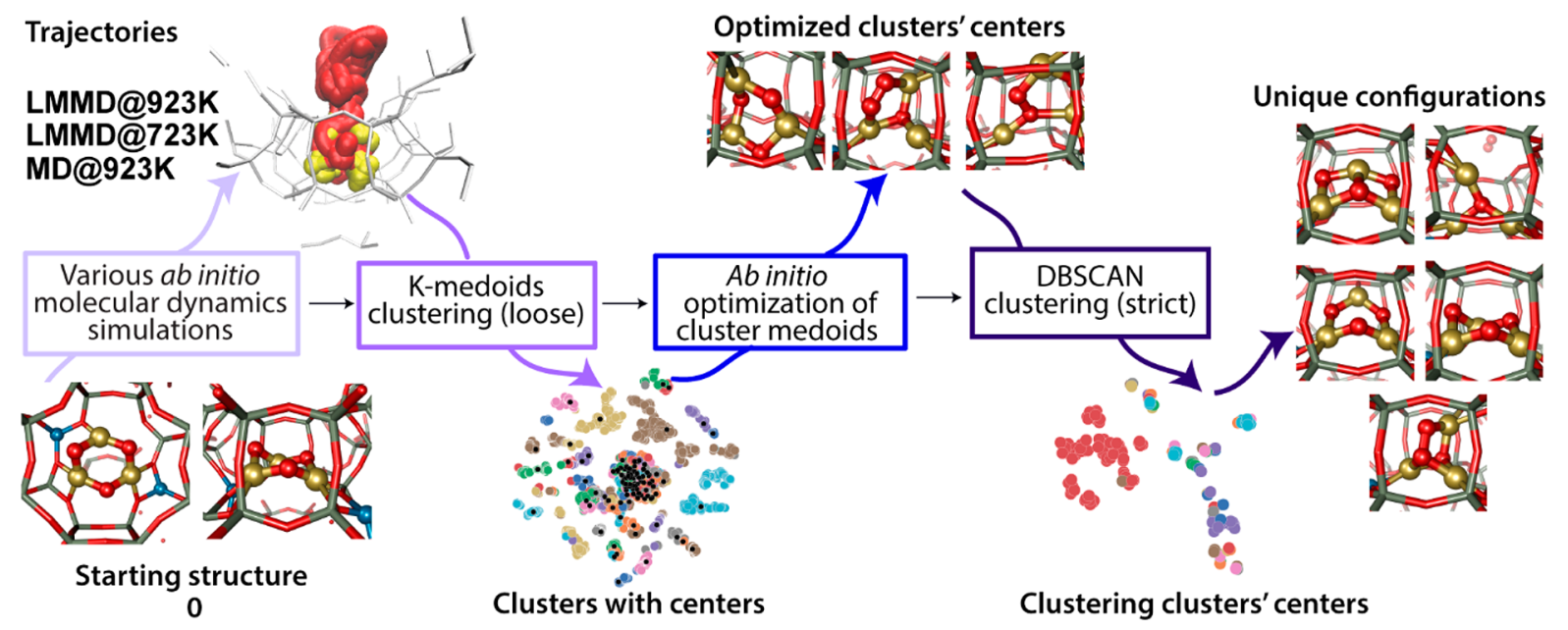

$\mathrm{CeO}_{2}$ where the reaction proceeds via two parallel routes in a small temperature region of $100-130{ }^{\circ} \mathrm{C} .{ }^{11}$ Dynamic surface reconstruction and the presence of metastable reactive ensembles on the catalyst surface have been proposed as the key factors behind the unique catalytic performance of boridebased materials. ${ }^{12}$ Reaction-induced reconstruction of supported $\mathrm{Pt}$ nanoclusters was shown to form specific reaction ensembles necessary for the efficient catalytic conversion of light alkanes. ${ }^{13}$ In zeolite catalysis, growing evidence is presented on the critical role of reaction-induced dynamics of the extraframework complexes. ${ }^{14}$ For example, the mobility of $\mathrm{Cu}$ ions in chabazite pores during the selective catalytic reduction was demonstrated in a combined experimental and computational study by Paolucci et al. ${ }^{15}$ The mobilization of the exchangeable $\mathrm{Cu}(\mathrm{I})$ site due to the interaction with the ammonia substrate allowed for their diffusion through the zeolite channels to form the catalytically active $\mathrm{Cu}$ (II) dimers.

Zeolites and related materials modified with metals/ nonmetals are promising catalysts as they possess specific reactive and oxidative environments induced by the framework-solid ligand. Their broad applicability in many processes results from the single-site nature of the catalytic sites and their well-defined structure, stability, and recyclability. ${ }^{16}$ Zeolites modified with $\mathrm{Cu}$ are capable of hydroxylating methane at low temperatures to produce methanol. ${ }^{17-20}$ Upon the activation with molecular $\mathrm{O}_{2}$, extraframework oxygenated copper species are formed in the zeolite channels capable of selectively activating the strong $\mathrm{C}-\mathrm{H}$ bond in methane. ${ }^{21}$ The ongoing debates on the nuclearity of the catalytically active centers have produced various hypothetical active sites, each supplied with spectroscopic and/or theoretical evidence. ${ }^{22}$ The situation here closely mimics that in oxo-copper clusters in methane monooxygenases (MMOs), where various structures of active sites were "proved" over the years, including a $\left[\mathrm{Cu}_{3}\left(\mu^{3}-\mathrm{O}\right)^{2+}\right]$ one. $^{21,23-28}$

The speciation of $\mathrm{Cu}$-oxo extraframework species relevant for methane oxofunctionalization was most extensively studied in ZSM-5 and MOR zeolites. Initial reactivity studies complemented by detailed UV-vis characterization attributed the unique methane activation activity of $\mathrm{Cu}$-zeolites to the presence of bis $(\mu$-oxo $)$ dicopper sites. ${ }^{29}$ This assignment has been later refined in a combined Raman spectroscopy and DFT study by Woertink et al., who concluded on the key role of mono( $\mu$-oxo $)$ dicopper in $\mathrm{Cu} / \mathrm{ZSM}-5$ zeolite for methane oxidation. ${ }^{30}$ The earlier characterization studies employed catalytic materials, in which only ca. $10 \%$ of the exchangeable $\mathrm{Cu}$ sites contributed to the methane activation activity. Grundner et $a l^{31}$ reported a $\mathrm{Cu} / \mathrm{MOR}$ catalyst, in which the majority of intrazeolite $\mathrm{Cu}$ was proposed to form the reactive ensembles. The catalysts were extensively characterized by EXAFS complemented by DFT-based $a b$ initio thermodynamic analysis, which revealed the presence of a cyclic chair-shaped $\left[\mathrm{Cu}_{3}(\mu-\mathrm{O})_{3}\right]^{2+}$ cluster. This configuration was later also proposed as the active site in other zeolite topologies. ${ }^{32-35}$ Further on, the increased stability and reactivity was shown for the clusters with higher nuclearity such as $\left[\mathrm{Cu}_{4} \mathrm{O}_{4}\right]^{2+}$ and $\left[\mathrm{Cu}_{5} \mathrm{O}_{5}\right]^{2+}{ }^{36}$ The reactivity and ensemble behavior of oxygenated alumina-supported $\mathrm{Cu}_{4} \mathrm{O}_{3}$ and $\mathrm{Cu}_{4} \mathrm{O}_{4}$ species were investigated by Sun et $a l^{37}$ It was proposed that the most stable configurations dominating the catalytic material are in fact inactive toward the oxidation of light alkanes. However, their isomerization into metastable configurations under the reaction conditions may give rise to pronounced catalytic reactivity.

Because the $\mathrm{Cu} / \mathrm{MOR}$ system operates at $\sim 475 \mathrm{~K}$, its catalytic site can be expected to show a high degree of fluxionality at the operando conditions, making the depiction of all its reactive conformers crucial for further mechanistic studies. Building a detailed ensemble of all the relevant structural isomers of a catalytic site is a challenging task, which, to the best of our knowledge, has not been addressed to date for zeolite catalysis.

To gain molecular-level insight into the catalytic mechanism and the nature of the catalytic sites where an ensemble of states can be pictured, various experimental and theoretical methods can be utilized. However, the possibility that only a minor part of the sites are catalytically active ${ }^{31}$ compromises most experimental methods which provide an averaged coarse picture of heavy atom alignment, as well as computational global-optimization approaches that aim to locate the most stable structures. ${ }^{38}$ The $a b$ initio molecular dynamics (aiMD), on the other hand, allow one to study the evolution of the preselected site under the catalytic conditions, with the drawback of being severely constrained to the starting structure. ${ }^{39}$ To overcome this limitation, a set of "biased" aiMD methods, including umbrella sampling, ${ }^{40}$ metadynam- 
ics $^{41}$ or thermodynamic integration, ${ }^{42}$ were designed, which, however, require a choice of the structural parameters determining the evolution of the system. ${ }^{43}$ For complex multibound systems like zeolite active sites this choice is highly nontrivial, and yet it is known that most chemical transformations proceed along the soft vibrational modes. ${ }^{44}$ Therefore, it is possible to utilize a low-mode following aiMD (LMMD) to simulate their evolution at time scales largely exceeding those of the actual molecular dynamics. The LMMD technique improves the sampling by following the lower vibrational modes, which narrows the explored conformational space to the physically meaningful configurations, making such an approach more affordable in combination with a higher level of theory.

Herein, we analyze the clusters which can be formed starting from the trimeric $\left[\mathrm{Cu}_{3}(\mu-\mathrm{O})_{3}\right]^{2+}$ site in MOR zeolite, for which chair conformation has been earlier proposed based on EXAFS data. To this end, we develop a new computational workflow enabling the exhaustive search of active site configurations (Scheme 1). Using this workflow we observe that while the copper triangle observed in EXAFS is indeed quite rigid, in the most stable conformations it is bound with a $\mu^{3}$-oxygen, similarly to one of the active sites hypothesized for MMO enzymes. The other two oxygens form a peroxo-unit, which, according to the thermodynamic analysis, is reversibly detachable as molecular oxygen leaving behind $\left[\mathrm{Cu}_{3}\left(\mu^{3}-\mathrm{O}\right)^{2+}\right.$ cluster.

The procedure of the exhaustive structural search (Scheme 1) starts with the exploration of the available configurational space for the extraframework species inside the zeolite pores via the $a b$ initio molecular dynamics (aiMD) simulations at the PBE-D3(BJ) level of theory. ${ }^{45-48}$ The resulting trajectories were analyzed using the loose clustering with $\mathrm{k}$-medoids algorithm to identify structurally distinct frames. As some of the clusters may lie at different slopes of the same valley, we optimized their centers and clustered them again using the density-based spatial clustering of applications with noise (DBSCAN) method, which is particularly suitable for the irregularly shaped clusters to determine the final unique configurations of the extraframework ensemble. The details of the computational methods and procedures are provided in the Supporting Information.

The key feature for the exhaustive sampling of the configurational space is the utilization of the low-mode following (also known as velocity softening), which encourages the system's evolution along the low vibrational modes. ${ }^{49}$ This approach was used in two distinct simulations carried out at high temperatures of $723 \mathrm{~K}$ (LMMD@723 K) and $923 \mathrm{~K}$ (LMMD@923 K) to improve the sampling. For comparison, the PES exploration using the conventional high-temperature aiMD simulation (MD@923 K) was also carried out.

Six trajectories of each simulation type were generated, providing 18 trajectories in total. Analyses of the root-meansquare displacements of the $\mathrm{Cu}_{3} \mathrm{O}_{3}$ fragment from the initial coordinates (RMSDs in Figure 1a) have shown that LMMD@ $723 \mathrm{~K}$ exhibited very limited variations in the oxo-copper cluster $\left(\mathrm{RMSD}_{\mathrm{MAX}}=1.1 \AA \mathrm{RMSD}_{\text {average }}=0.5 \AA\right)$, two out of six MD@923 K trajectories exceeded RMSD of $2 \AA$ $\left(\mathrm{RMSD}_{\mathrm{MAX}}=4.4 \AA, \mathrm{RMSD}_{\text {average }}=1.0 \AA\right)$, and LMMD@ $923 \mathrm{~K}$ demonstrated the highest variation with four out of six trajectories exceeding RMSD of $2 \AA\left(\mathrm{RMSD}_{\mathrm{MAX}}=9.2 \AA\right.$ and $\left.\mathrm{RMSD}_{\text {average }}=2.4 \AA\right)$. Thus, both elevated temperature and the low-mode following approach are crucial for an extensive

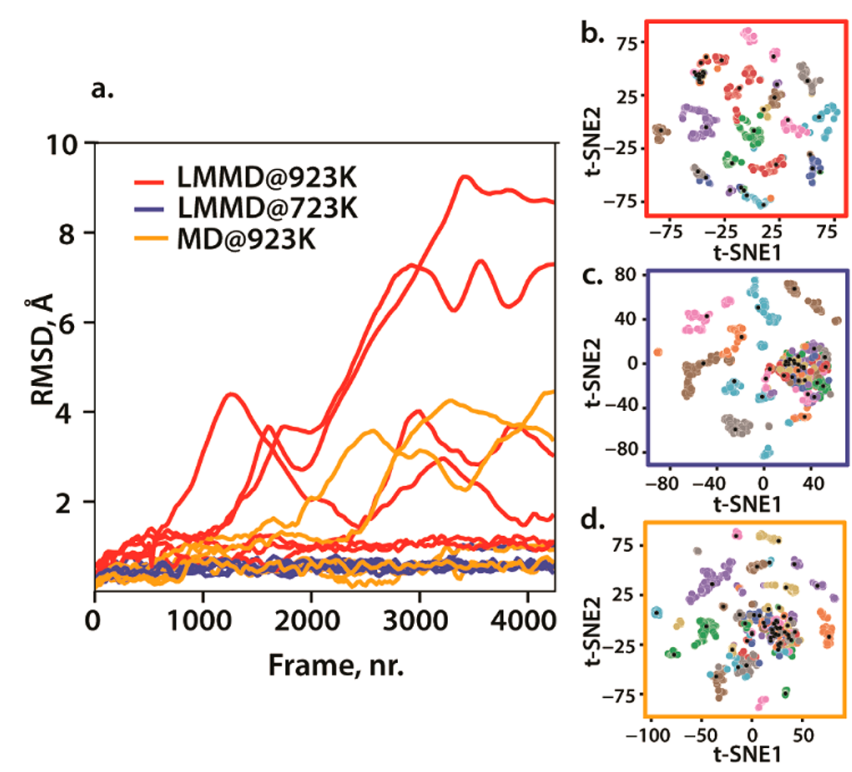

Figure 1. (a) RMSD of the different simulation trajectories for $\left[\mathrm{Cu}_{3} \mathrm{O}_{3}\right]^{2+}$ in MOR. The results of the $k$-medoids clustering of LMMD@923 K (b, the highest silhouette score is 0.41 at 44 clusters), LMMD@723 K (c, the highest silhouette score is 0.34 at 36 clusters), $\mathrm{MD} @ 923 \mathrm{~K}$ (d, the highest silhouette score is 0.31 at 66 clusters), projected to $2 \mathrm{D}$ plane via $\mathrm{t}$-SNE algorithm. Cluster centers are denoted with black dots.

sampling of the atomic configurations for a zeolite-bound oxocopper cluster; note, however, that two of the red trajectories (LMMD@923 K) did not exhibit large structural variations, probably because the randomly selected low modes along which the system was dragged did not lead to the active site evolution. This result highlights the necessity of running several elevated-temperature LMMDs in order to sufficiently sample the configurational space; as we will see later, only this approach allowed us to locate the most stable $\left[\mathrm{Cu}_{3} \mathrm{O}_{3}\right]^{2+}$ configurations.

Figure $1 \mathrm{~b}-\mathrm{d}$ illustrates the clustering of each type of trajectories to identify the unique groups of clusters using an unsupervised machine learning $k$-medoids algorithm with the "silhouette" index for choosing $k^{50-52}$ Clustering algorithms can capture the differences and similarities among the structures and show the relationships in the underlying properties. Among the different clustering approaches, we have chosen the $k$-medoids method. ${ }^{52}$ This approach constructs (hyper)spherical clusters and naturally provides coordinates of the medoid points (i.e., the structures of "median catalytic sites" for each cluster), which correspond to real data points (denoted as "cluster centers" henceforth).

Initially, we have clustered LMMD@723 K, MD@923 K, and LMMD@923 K sets of trajectories separately, using every 50th frame (we use a time step of $0.5 \mathrm{fs}$, so time lags between the clustered structures are $25 \mathrm{fs}$ ). It gave 1020 frames of each type of trajectory. This procedure resulted in the clusters illustrated in Figure $1 \mathrm{~b}-\mathrm{d}$ using the $\mathrm{t}$-SNE ( $\mathrm{t}$-distributed stochastic neighbor embedding) dimensionality reduction technique, which allows projecting the high-dimensional data (6 atoms of the $\left[\mathrm{Cu}_{3} \mathrm{O}_{3}\right]^{2+}$ cluster in $3 \mathrm{D}$ give dimensionality of 18D) onto two dimensions. ${ }^{53}$ The silhouette index reflecting the similarity of each data point with its own compared to all other clusters was used to determine the optimal number of clusters. Relatively low, yet sufficient for clustering analysis, 
values of the silhouette scores for all trajectories indicate a weak separation of the individual clusters due to the high mobility and configurational freedom of the extraframework species at the simulation temperatures. More detailed information on the silhouette score analysis is given in Figure S1a-d. The silhouette score assessment for LMMD@923 K gave 44 distinct clusters (Figure 1b), whereas 66 and 36 clusters were obtained from MD@923 K and LMMD@723 K trajectories, respectively.

Because some of the clusters could lie at different slopes of the same valley, we have optimized structures of all 146 located cluster centers at the same level of theory as above, now considering two possible multiplicities, triplet $(S=3 / 2)$ and singlet $(S=1 / 2)$, for each cluster center. ${ }^{34}$ Figure 2

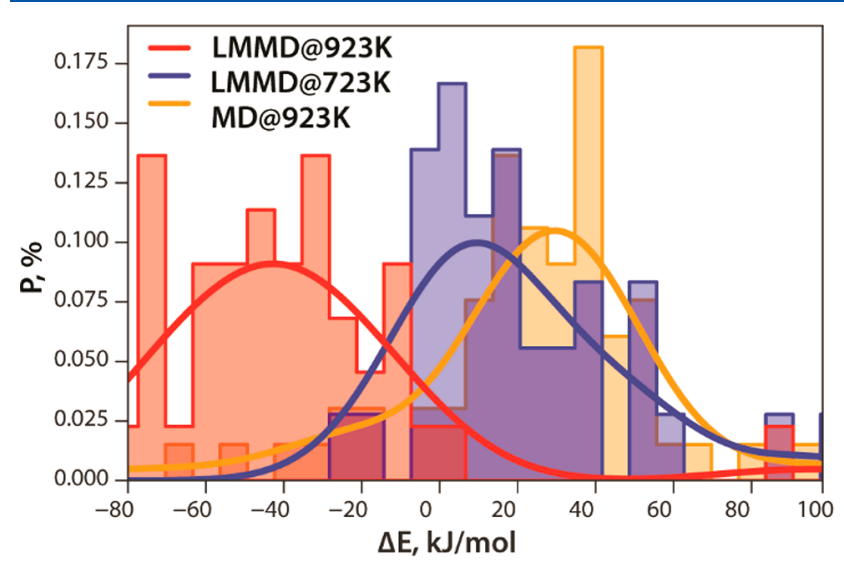

Figure 2. Energy distribution of the optimized clusters' centers obtained via $k$-medoids clustering procedure. $P$ stands for the probability, and $\Delta E$ is the energy difference between the isomer and the initial configuration.

summarizes the distribution of the relative energies $(\Delta E)$ of the optimized clusters' centers compared to the initial cyclicchair $\left[\mathrm{Cu}_{3}(\mu \text {-O })_{3}\right]^{2+}$ configuration which was assigned relative energy of $0 \mathrm{~kJ} \mathrm{~mol}^{-1}$. Each subset of trajectories has been normalized independently, and a Gaussian kernel density estimate was used to smoothen the distribution. ${ }^{54} 60$ out of 146 identified cluster centers are lower in energy than the initial configuration.

Notably, energy distributions show that only LMMD@923 $\mathrm{K}$ produces structures which are on average more stable than the initial one. Although the LMMD@723 K set of trajectories has the lowest RMSD deviation from the staring configuration (Figure 1a), it still locates structurally distinct isomers with negative relative energies. Comparison of LMMD@723 K and MD@923 K energy distributions highlights the higher efficiency of the low-mode-following procedure in locating low-energy configurations relative to the brute-force temperature elevation.

Geometry optimization resulted in the convergence of the cluster centers into the corresponding minima. These were clustered again using the DBSCAN ${ }^{55}$ method, which is good for finding dense misshaped clusters. 146 initial cluster centers were distributed into 47 unique clusters; to evade confusion, we denote the initial 146 clusters "first-gen clusters", and the refined 47 ones as the "second-gen clusters". The energetics of the second-gen clusters was refined through the single-point calculations using a hybrid functional (PBE0). Figure S4 shows a general consistency between the PBE0 and PBE, with lower favorability for $\left[\mathrm{Cu}_{3}\left(\mu^{3}-\mathrm{O}\right)\right]^{2+}$ and molecular $\mathrm{O}_{2}$ bound to it at the PBE0 level of theory. Detailed properties of the second-gen clusters, their relation to the first-gen ones, and the initial structures are outlined in Figure S3 and Table S1. The secondgen clusters significantly vary in size. Whereas some of them are represented by single configurations (e.g., 2, 4, 6, 9, 10, 12$32,34,36$, and $38-47$ ), the others include multiple structures with similar local geometries but different energies (e.g., 1, 3, 5, $7,8,11,33,35$, and 37).

Our procedure identified four distinct $\left[\mathrm{Cu}_{3} \mathrm{O}_{3}\right]^{2+}$ families each forming broad ensembles of configurations. The lowestenergy family I (second-gen clusters $1-13$, gray in Figure 3) features a $\mathrm{Cu}_{3}\left(\mu^{3}-\mathrm{O}\right)$ core coordinated to a peroxo ligand with two $\mathrm{Cu}$ ions, which adapt either a distorted square-planar or seesaw coordinational environments. These structures were predominantly found with the LMMD@923 K search producing 581 frames with $\left[\mathrm{Cu}_{3}\left(\mu^{3}-\mathrm{O}\right)(\mathrm{O}-\mathrm{O})\right]^{2+}$ structures, whereas the LMMD@723 K and the MD@923 K give 0 and 17 frames of this type, respectively. The second family II of configurations (second-gen clusters 14-16, orange in Figure 3) features a peroxo-ligand linking $\mathrm{Cu}_{2} \mathrm{O}$ and $\mathrm{Cu}$ moieties. These configurations are on average less stable than the peroxo-species of family I. They are observed in just 24 and 86 frames of the LMMD@923 K and LMMD@723 K trajectories, respectively. The third family III of structures (second-gen clusters 17-32, blue in Figure 3) represents formally reduced $\mathrm{Cu}$ trimers $\left[\mathrm{Cu}_{3}\left(\mu^{3}-\mathrm{O}\right)\right]^{2+}$ in the MOR sidepocket with molecular $\mathrm{O}_{2}$ physisorbed within the main channel of MOR or weakly bound to one of the $\mathrm{Cu}$ centers. Such configurations were found only in the high-temperature runs with 415 and 150 frames, respectively, in LMMD@923 K and MD@923 K trajectories. The fourth family IV (second-gen clusters 33-47, magenta in Figure 3) encompasses $\mathrm{Cu}$-oxo trimers corresponding to $(\mathbf{I V a})\left[\mathrm{Cu}_{3}\left(\mu^{2}-\mathrm{O}\right)_{3}\right]^{2+}$ isomers similar to the starting configuration and (IVb) $\left[\mathrm{Cu}_{3}\left(\mu^{2}-\mathrm{O}\right)_{2}\left(\mu^{3}-\mathrm{O}\right)\right]^{2+}$ species in an "envelope" configuration with two $\mathrm{Cu}$ ions in a distorted planar coordination and one $\mathrm{Cu}$ ion in a $\mathrm{T}$-shaped coordination. Type IV structures were found only in LMMD@ $723 \mathrm{~K}$ and $\mathrm{MD} @ 923 \mathrm{~K}$ trajectories. The $\mathrm{Cu}_{3}\left(\mu^{2}-\mathrm{O}\right)_{3}{ }^{2+}$ clusters (IVa) predominantly discussed in the prior literature were found in 152 and 93 frames of the respective simulations. The alternative IVb configurations dominated the LMMD@723 K and MD@923 K trajectories, giving 782 and 760 frames, respectively. It is worth noting that these configurations are at least $50 \mathrm{~kJ} \mathrm{~mol}^{-1}$ less stable than those belonging to type I structures.

Therefore, our analysis shows that a single stoichiometry of an oxygenated copper cluster can form a wide variety of lowlying configurations that were not proposed earlier. The lowmode following approach introduced in this study uniquely allows identifying such configurations. For $\mathrm{Cu} / \mathrm{MOR}$, the LMMD@923 K search of the configurational space predominantly gave the structures featuring an adsorbed $\mathrm{O}-\mathrm{O}$ peroxo or molecular $\mathrm{O}_{2}$ on a stable $\left[\mathrm{Cu}_{3}\left(\mu^{3}-\mathrm{O}\right)\right]^{2+}$ core, resembling the trinuclear copper active site proposed for methane monooxygenases. ${ }^{28}$

Next, we carried out an ab initio thermodynamics analysis (aiTA) to investigate the relative stabilities of the obtained isomers under reactive conditions. The results are summarized in Figure 4 presenting the Gibbs free energies of optimized $\left[\mathrm{Cu}_{3} \mathrm{O}_{3}\right]^{2+}$ isomers, calculated from their reduced counterpart $\left[\mathrm{Cu}_{3} \mathrm{O}\right]^{2+}$, as a function of oxygen chemical potential, $\mu(\mathrm{O})$ (for further information see section 1 in the Supporting 


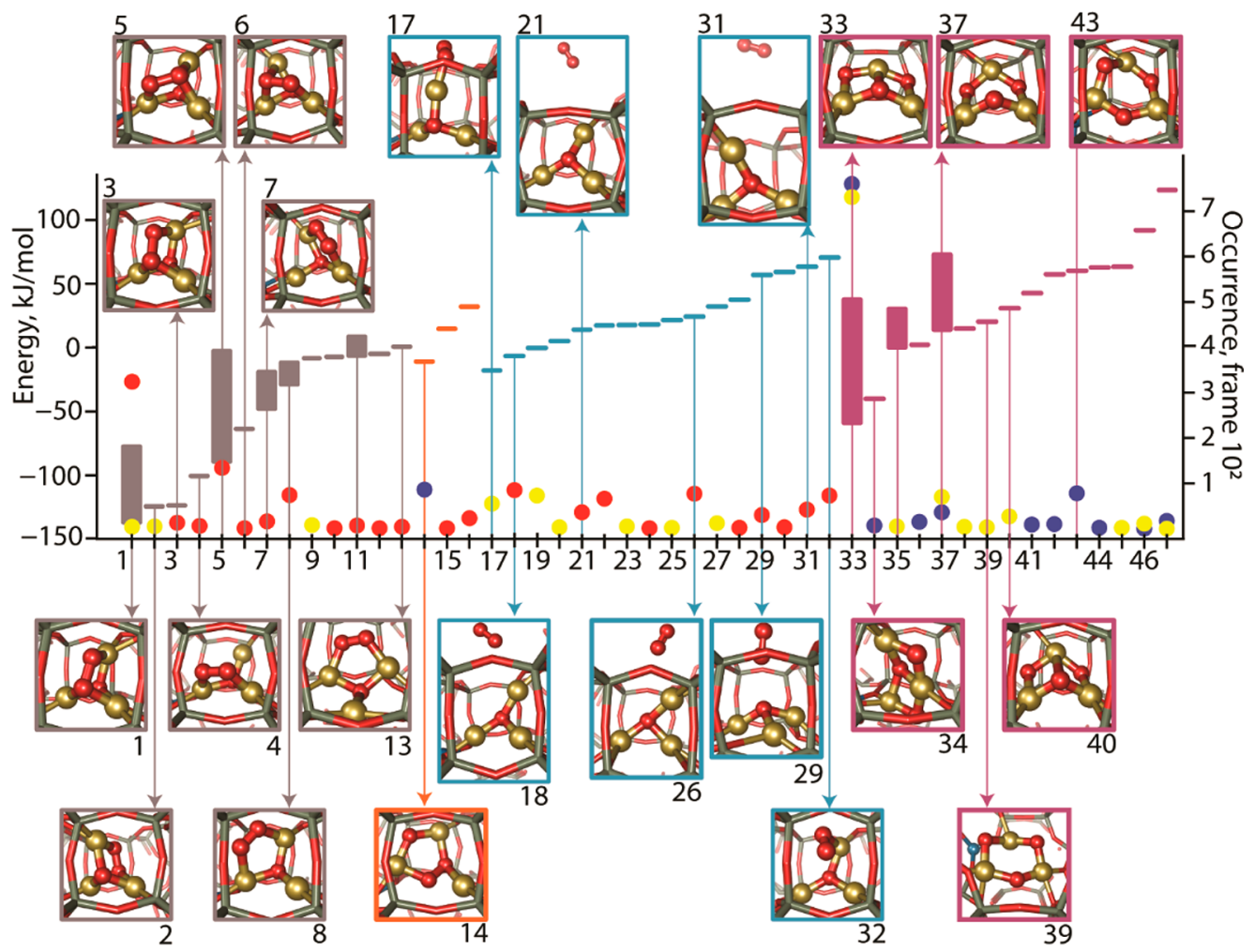

- nr. frames from LMMD@923K_nr.frames from LMMD@723K nr. frames from MD@923K

Figure 3. Description of the second-generation clusters obtained from the optimized first-generation clusters' centers. The geometries were optimized at the PBE-D3(BJ) level of theory, and the energies were improved with single point calculations at PBE0-D3(BJ). For each cluster, the range of energies of the residing configurations (bars of - left axis) is given with the connected insets depicting the optimized local geometry of the lowest-lying extraframework $\mathrm{Cu}$ trimer structure. The occurrence of the structures $(\boldsymbol{\bullet}$, right axis) belonging to the cluster is shown as the number of frames from the initial aiMD trajectories with respect to the first clustering procedure. The contribution of each aiMD type into each second-generation cluster is differentiated by color with red, blue, and yellow circles corresponding to LMMD@923 K, LMMD@723 K, and MD@ $923 \mathrm{~K}$ trajectories, respectively. For a complete set of structures, we refer readers to Table S1.

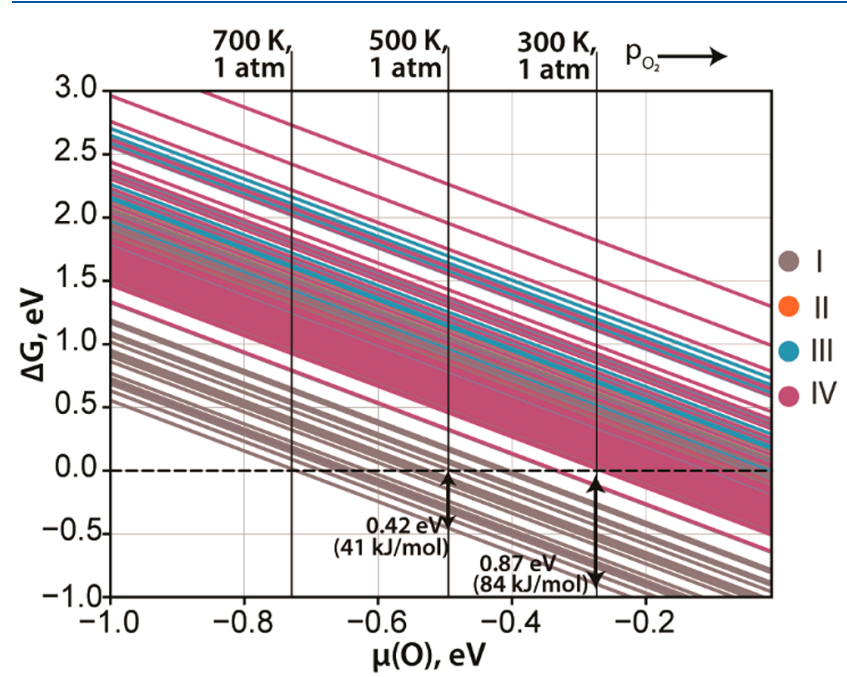

Figure 4. Free energies of formation of $\mathrm{Cu}_{3} \mathrm{O}_{3}{ }^{2+}$ isomers from the partially reduced $\mathrm{Cu}_{3} \mathrm{O}^{2+}$ cation in MOR as a function of oxygen chemical potential. The color code is used to differentiate the different cluster families.

Information, $A b$ Initio Thermodynamics Analysis). The electronic structures of all optimized clusters were analyzed in terms of Hirshfeld charges and spin densities, which did not reveal any correlation with the relative stability (Figure S5, S6).
The lowest-energy structure of $\left[\mathrm{Cu}_{3} \mathrm{O}_{3}\right]^{2+}$ contains a $\mathrm{Cu}_{3}\left(\mu^{3}-\mathrm{O}\right)$ moiety similar to that found in the methane monooxygenase ${ }^{28}$ with an $\mathrm{O}_{2}$ molecule chemisorbed on it. Notably, a deoxygenated $\mathrm{Cu}_{3}\left(\mu^{3}-\mathrm{O}\right) / \mathrm{MOR}$ system appears to be very stable during LMMD@923 $\mathrm{K}$ and does not change its structure during the $\sim 5 \mathrm{ps}$ run. This behavior drastically differs from that of its $\mathrm{Cu}_{3} \mathrm{O}_{3}$ counterpart, indicating that the oxidation strongly increases the flexibility of the extraframework multinuclear cation.

On the basis of our calculations, $\mathrm{Cu}_{3}\left(\mu^{3}-\mathrm{O}\right)$ can be expected to coexist with the most stable found $\mathrm{Cu}_{3}\left(\mu^{3}-\mathrm{O}\right)\left(\eta-\mathrm{O}_{2}\right)$ cluster at $\mu(\mathrm{O}) \approx-0.71 \mathrm{eV}$, corresponding to approximately $700 \mathrm{~K}$ at 1 atm of $\mathrm{O}_{2}$ (catalyst activation). At lower $\mu(\mathrm{O})$, that is at higher temperatures and/or lower oxygen pressure, the autoreduction of the extraframework cluster to $\mathrm{Cu}_{3}\left(\mu^{3}-\mathrm{O}\right)$ should take place. However, under the conditions of the catalyst activation, ${ }^{19}$ the $\mathrm{Cu}_{3}\left(\mu^{3}-\mathrm{O}\right)\left(\eta-\mathrm{O}_{2}\right)$ configurations become more energetically accessible. Specifically, we find that the $\mathrm{Cu}_{3}\left(\mu^{3}-\mathrm{O}\right)\left(\eta-\mathrm{O}_{2}\right)$ is $40-80 \mathrm{~kJ} / \mathrm{mol}$ more thermodynamically stable than its reduced counterpart in the temperature range of 500-300 $\mathrm{K}$ corresponding to the temperatures of the catalytic methane oxidation or steaming (Figure 4).

In this work we analyzed the clusters which can be formed starting from the trimeric $\left[\mathrm{Cu}_{3}(\mu-\mathrm{O})_{3}\right]^{2+}$ site in MOR zeolite, for which chair conformation has been earlier proposed based on EXAFS data. To this end, we develop a new computational 
workflow enabling the exhaustive search of active site configurations (Scheme 1). Our analysis explores the structural complexity and the highly dynamic nature of oxygenated multinuclear extraframework species in zeolite pores and emphasizes the need for new expert bias-free computational approaches to address the structural problem of zeolite catalysis.

The proposed approach for the exhaustive configurational search addresses the structural problem associated with the identification of the extraframework ensembles in zeolite catalysis in several steps. First, we sample the neighboring regions of the PES around a reference structure-the cyclic chair-shaped $\left[\mathrm{Cu}_{3}(\mu \text {-O })_{3}\right]^{2+}$ cluster confined in the side pocket of mordenite in this work-by dragging the system along the low modes in the LMMD simulations. This promotes an enhanced sampling that gives the atomic configurations reflecting the surrounding basins. These can be viewed as an ensemble of structures occurring under reactive conditions. Then, we cluster the LMMD trajectories using $k$-medoids to find distinguishable clouds of points corresponding to different clusters. Because some of the clusters can belong to the same energy valley, an additional step of geometry optimization of the cluster centers following the second clustering using DBSCAN is introduced.

For the $\left[\mathrm{Cu}_{3} \mathrm{O}_{3}\right]^{2+}$ cation in MOR, this procedure identified 47 distinct sites corresponding to four archetypes, among which highly stable new configurations previously not considered in experimental or computational studies were found. Finally, the thermodynamic favorability of the obtained conformations under reactive conditions indicated a link between the oxygen chemical potential and the occurrence of structures belonging to different families.

\section{ASSOCIATED CONTENT}

\section{SI Supporting Information}

The Supporting Information is available free of charge at https://pubs.acs.org/doi/10.1021/acs.jpclett.1c03288.

Computational details and discussion of $k$-medoids clustering, DBSCAN clustering, and methane activation (PDF)

Optimized atomic coordinates, total energies (a.u.), relative stabilities $(\mathrm{kJ} / \mathrm{mol})$, and occurrence in $\mathrm{MD}$ trajectories of the periodic structures from the secondgen clusters (XYZ)

\section{AUTHOR INFORMATION}

\section{Corresponding Author}

Evgeny A. Pidko - Inorganic Systems Engineering (ISE), Department of Chemical Engineering, Delft University of Technology, 2629 HZ Delft, The Netherlands; 이이.org/ 0000-0001-9242-9901; Email: e.a.pidko@tudelft.nl

\section{Authors}

Elena V. Khramenkova - Inorganic Systems Engineering (ISE), Department of Chemical Engineering, Delft University of Technology, 2629 HZ Delft, The Netherlands

Michael G. Medvedev - Zelinsky Institute of Organic Chemistry RAS, Moscow 119991, Russia; ㅇorcid.org/ 0000-0001-7070-4052

Guanna Li - Biobased Chemistry \& Technology, Wageningen University \& Research, 6708 PB Wageningen, The Netherlands; Organic Chemistry, Wageningen University \&
Research, 6708 PB Wageningen, The Netherlands; (1) orcid.org/0000-0003-3031-8119

Complete contact information is available at:

https://pubs.acs.org/10.1021/acs.jpclett.1c03288

\section{Notes}

The authors declare no competing financial interest.

\section{ACKNOWLEDGMENTS}

This project was enabled by the financial support from the European Research Council (ERC) under the European Union's Horizon 2020 research and innovation programme (Grant Agreement No. 725686). The use of supercomputer facilities was sponsored by NWO Domain Science.

\section{REFERENCES}

(1) Shetty, M.; Walton, A.; Gathmann, S. R.; Ardagh, M. A.; Gopeesingh, J.; Resasco, J.; Birol, T.; Zhang, Q.; Tsapatsis, M.; Vlachos, D. G.; Christopher, P.; Frisbie, C. D.; Abdelrahman, O. A.; Dauenhauer, P. J. The Catalytic Mechanics of Dynamic Surfaces: Stimulating Methods for Promoting Catalytic Resonance. ACS Catal. 2020, 10 (21), 12666-12695.

(2) Bruix, A.; Margraf, J. T.; Andersen, M.; Reuter, K. FirstPrinciples-Based Multiscale Modelling of Heterogeneous Catalysis. Nat. Catal. 2019, 2 (8), 659-670.

(3) Warshel, A.; Bora, R. P. Perspective: Defining and Quantifying the Role of Dynamics in Enzyme Catalysis. J. Chem. Phys. 2016, 144 (18), 180901.

(4) Ananikov, V. P.; Beletskaya, I. P. Toward the Ideal Catalyst: From Atomic Centers to a "Cocktail" of Catalysts. Organometallics 2012, 31 (5), 1595-1604.

(5) Galushko, A. S.; Gordeev, E. G.; Kashin, A. S.; Zubavichus, Y. V.; Ananikov, V. P. Visualization of Catalyst Dynamics and Development of a Practical Procedure to Study Complex "Cocktail"-Type Catalytic Systems. Faraday Discuss. 2021, 229 (0), 458-474.

(6) Zhang, Z.; Zandkarimi, B.; Alexandrova, A. N. Ensembles of Metastable States Govern Heterogeneous Catalysis on Dynamic Interfaces. Acc. Chem. Res. 2020, 53 (2), 447-458.

(7) Medvedev, M. G.; Zeifman, A. A.; Novikov, F. N.; Bushmarinov, I. S.; Stroganov, O. V.; Titov, I. Y.; Chilov, G. G.; Svitanko, I. V. Quantifying Possible Routes for SpnF-Catalyzed Formal Diels-Alder Cycloaddition. J. Am. Chem. Soc. 2017, 139 (11), 3942-3945.

(8) Yaremenko, I. A.; Radulov, P. S.; Medvedev, M. G.; Krivoshchapov, N. V.; Belyakova, Y. Y.; Korlyukov, A. A.; Ilovaisky, A. I.; Terent'ev, A. O.; Alabugin, I. V. How to Build Rigid OxygenRich Tricyclic Heterocycles from Triketones and Hydrogen Peroxide: Control of Dynamic Covalent Chemistry with Inverse $\alpha$-Effect. J. Am. Chem. Soc. 2020, 142 (34), 14588-14607.

(9) Cruchter, T.; Medvedev, M. G.; Shen, X.; Mietke, T.; Harms, K.; Marsch, M.; Meggers, E. Asymmetric Nucleophilic Catalysis with an Octahedral Chiral-at-Metal Iridium(III) Complex. ACS Catal. 2017, 7 (8), 5151-5162.

(10) Larionov, V. A.; Savel'yeva, T. F.; Medvedev, M. G.; Stoletova, N. V.; Smol'yakov, A. F.; Gugkaeva, Z. T.; Cruchter, T.; Maleev, V. I. The Selective N-Functionalization of Indoles via Aza-Michael Addition in the Ligand Sphere of a Chiral Nickel(II) Complex: Asymmetric Synthesis of (S)-1H-Indole-Alanine Derivatives. Eur. J. Org. Chem. 2019, 2019 (22), 3699-3703.

(11) Zhang, X.; Deng, Y. Q.; Tian, P.; Shang, H.; Xu, J.; Han, Y. F. Dynamic Active Sites over Binary Oxide Catalysts: In Situ/Operando Spectroscopic Study of Low-Temperature CO Oxidation over MnOxCeO2 Catalysts. Appl. Catal., B 2016, 191, 179-191.

(12) Zhang, Z.; Cui, Z.-H.; Jimenez-Izal, E.; Sautet, P.; Alexandrova, A. N. Hydrogen Evolution on Restructured B-Rich WB: Metastable Surface States and Isolated Active Sites. ACS Catal. 2020, 10 (23), 13867-13877. 
(13) Sun, G.; Fuller, J. T.; Alexandrova, A. N.; Sautet, P. Global Activity Search Uncovers Reaction Induced Concomitant Catalyst Restructuring for Alkane Dissociation on Model Pt Catalysts. ACS Catal. 2021, 11 (3), 1877-1885.

(14) Krishna, S. H.; Jones, C. B.; Gounder, R. Dynamic Interconversion of Metal Active Site Ensembles in Zeolite Catalysis. Annu. Rev. Chem. Biomol. Eng. 2021, 12 (1), 115-136.

(15) Paolucci, C.; Khurana, I.; Parekh, A. A.; Li, S.; Shih, A. J.; Li, H.; Di Iorio, J. R.; Albarracin-Caballero, J. D.; Yezerets, A.; Miller, J. T.; Delgass, W. N.; Ribeiro, F. H.; Schneider, W. F.; Gounder, R. Dynamic Multinuclear Sites Formed by Mobilized Copper Ions in NOx Selective Catalytic Reduction. Science (Washington, DC, U. S.) 2017, 357 (6354), 898-903.

(16) Pidko, E. A.; Hensen, E. J. M.; Van Santen, R. A. SelfOrganization of Extraframework Cations in Zeolites. Proceedings of the Royal Society A: Mathematical, Physical and Engineering Sciences 2012, 468, 2070-2086, DOI: 10.1098/rspa.2012.0057.

(17) Dinh, K. T.; Sullivan, M. M.; Serna, P.; Meyer, R. J.; Dincă, M.; Román-Leshkov, Y. Viewpoint on the Partial Oxidation of Methane to Methanol Using $\mathrm{Cu}$ - and Fe-Exchanged Zeolites. ACS Catal. 2018, 8 (9), 8306-8313.

(18) Mahyuddin, M. H.; Shiota, Y.; Yoshizawa, K. Methane Selective Oxidation to Methanol by Metal-Exchanged Zeolites: A Review of Active Sites and Their Reactivity. Catal. Sci. Technol. 2019, 9 (8), 1744-1768.

(19) Newton, M. A.; Knorpp, A. J.; Sushkevich, V. L.; Palagin, D.; van Bokhoven, J. A. Active Sites and Mechanisms in the Direct Conversion of Methane to Methanol Using $\mathrm{Cu}$ in Zeolitic Hosts: A Critical Examination. Chem. Soc. Rev. 2020, 49 (5), 1449-1486.

(20) Sushkevich, V. L.; van Bokhoven, J. A. Methane-to-Methanol: Activity Descriptors in Copper-Exchanged Zeolites for the Rational Design of Materials. ACS Catal. 2019, 9 (7), 6293-6304.

(21) Snyder, B. E. R.; Bols, M. L.; Schoonheydt, R. A.; Sels, B. F.; Solomon, E. I. Iron and Copper Active Sites in Zeolites and Their Correlation to Metalloenzymes. Chem. Rev. 2018, 118 (5), 27182768.

(22) Ravi, M.; Sushkevich, V. L.; Knorpp, A. J.; Newton, M. A.; Palagin, D.; Pinar, A. B.; Ranocchiari, M.; van Bokhoven, J. A. Misconceptions and Challenges in Methane-to-Methanol over Transition-Metal-Exchanged Zeolites. Nat. Catal. 2019, 2 (6), 485494.

(23) Chen, Y.-H.; Wu, C.-Q.; Sung, P.-H.; Chan, S. I.; Chen, P. P.-Y. Turnover of a Methane Oxidation Tricopper Cluster Catalyst: Implications for the Mechanism of the Particulate Methane Monooxygenase (PMMO). ChemCatChem 2020, 12 (11), 30883096.

(24) Ross, M. O.; MacMillan, F.; Wang, J.; Nisthal, A.; Lawton, T. J.; Olafson, B. D.; Mayo, S. L.; Rosenzweig, A. C.; Hoffman, B. M. Particulate Methane Monooxygenase Contains Only Mononuclear Copper Centers. Science 2019, 364 (6440), 566-570.

(25) Solomon, E. I.; Heppner, D. E.; Johnston, E. M.; Ginsbach, J. W.; Cirera, J.; Qayyum, M.; Kieber-Emmons, M. T.; Kjaergaard, C. H.; Hadt, R. G.; Tian, L. Copper Active Sites in Biology. Chem. Rev. 2014, 114 (7), 3659-3853.

(26) Prieto, G.; Zečević, J.; Friedrich, H.; De Jong, K. P.; De Jongh, P. E. Towards Stable Catalysts by Controlling Collective Properties of Supported Metal Nanoparticles. Nat. Mater. 2013, 12 (1), 34-39.

(27) Rosenzweig, A. C. The Metal Centres of Particulate Methane Mono-Oxygenase. Biochem. Soc. Trans. 2008, 36 (6), 1134-1137.

(28) Chan, S. I.; Wang, V. C. C.; Lai, J. C. H.; Yu, S. S. F.; Chen, P. P. Y.; Chen, K. H. C.; Chen, C. L.; Chan, M. K. Redox Potentiometry Studies of Particulate Methane Monooxygenase: Support for a Trinuclear Copper Cluster Active Site. Angew. Chem., Int. Ed. 2007, 46 (12), 1992-1994.

(29) Groothaert, M. H.; Smeets, P. J.; Sels, B. F.; Jacobs, P. A.; Schoonheydt, R. A. Selective Oxidation of Methane by the Bis $(\mathrm{Mu}-$ Oxo)Dicopper Core Stabilized on ZSM-5 and Mordenite Zeolites. J. Am. Chem. Soc. 2005, 127 (5), 1394-1395.
(30) Woertink, J. S.; Smeets, P. J.; Groothaert, M. H.; Vance, M. A.; Sels, B. F.; Schoonheydt, R. A.; Solomon, E. I. A [Cu2O $] 2+$ Core in Cu-ZSM-5, the Active Site in the Oxidation of Methane to Methanol. Proc. Natl. Acad. Sci. U. S. A. 2009, 106 (45), 18908-18913.

(31) Grundner, S.; Markovits, M. A. C.; Li, G.; Tromp, M.; Pidko, E. A.; Hensen, E. J. M.; Jentys, A.; Sanchez-Sanchez, M.; Lercher, J. A. Single-Site Trinuclear Copper Oxygen Clusters in Mordenite for Selective Conversion of Methane to Methanol. Nat. Commun. 2015, 6 (May), 1-9.

(32) Ikuno, T.; Grundner, S.; Jentys, A.; Li, G.; Pidko, E.; Fulton, J.; Sanchez-Sanchez, M.; Lercher, J. A. Formation of Active $\mathrm{Cu}$-Oxo Clusters for Methane Oxidation in Cu-Exchanged Mordenite. J. Phys. Chem. C 2019, 123 (14), 8759-8769.

(33) Zheng, J.; Lee, I.; Khramenkova, E.; Wang, M.; Peng, B.; Gutiérrez, O. Y.; Fulton, J. L.; Camaioni, D. M.; Khare, R.; Jentys, A.; Haller, G. L.; Pidko, E. A.; Sanchez-Sanchez, M.; Lercher, J. A. Importance of Methane Chemical Potential for Its Conversion to Methanol on Cu-Exchanged Mordenite. Chem. - Eur. J. 2020, 26 (34), $7563-7567$

(34) Li, G.; Vassilev, P.; Sanchez-Sanchez, M.; Lercher, J. A.; Hensen, E. J. M.; Pidko, E. A. Stability and Reactivity of Copper OxoClusters in ZSM-5 Zeolite for Selective Methane Oxidation to Methanol. J. Catal. 2016, 338, 305-312.

(35) Vogiatzis, K. D.; Li, G.; Hensen, E. J. M.; Gagliardi, L.; Pidko, E. A. Electronic Structure of the $[\mathrm{Cu} 3(\mu-\mathrm{O}) 3] 2+$ Cluster in Mordenite Zeolite and Its Effects on the Methane to Methanol Oxidation. J. Phys. Chem. C 2017, 121 (40), 22295-22302.

(36) Palagin, D.; Knorpp, A. J.; Pinar, A. B.; Ranocchiari, M.; van Bokhoven, J. A. Assessing the Relative Stability of Copper Oxide Clusters as Active Sites of a CuMOR Zeolite for Methane to Methanol Conversion: Size Matters? Nanoscale 2017, 9 (3), 11441153.

(37) Sun, G.; Alexandrova, A. N.; Sautet, P. Structural Rearrangements of Subnanometer $\mathrm{Cu}$ Oxide Clusters Govern Catalytic Oxidation. ACS Catal. 2020, 10 (9), 5309-5317.

(38) Zhang, Z.; Jimenez-Izal, E.; Hermans, I.; Alexandrova, A. N. Dynamic Phase Diagram of Catalytic Surface of Hexagonal Boron Nitride under Conditions of Oxidative Dehydrogenation of Propane. J. Phys. Chem. Lett. 2019, 10 (1), 20-25.

(39) Imaoka, T.; Toyonaga, T.; Morita, M.; Haruta, N.; Yamamoto, K. Isomerizations of a Pt4 Cluster Revealed by Spatiotemporal Microscopic Analysis. Chem. Commun. 2019, 55 (33), 4753-4756.

(40) Kästner, J. Umbrella Sampling. Wiley Interdiscip. Rev. Comput. Mol. Sci. 2011, 1 (6), 932-942.

(41) Barducci, A.; Bonomi, M.; Parrinello, M. Metadynamics. Wiley Interdiscip. Rev.: Comput. Mol. Sci. 2011, 1 (5), 826-843.

(42) Abrams, C.; Bussi, G. Enhanced Sampling in Molecular Dynamics Using Metadynamics, Replica-Exchange, and TemperatureAcceleration. Entropy 2014, 16 (1), 163-199.

(43) Martínez-Suárez, L.; Frenzel, J.; Marx, D.; Meyer, B. Tuning the Reactivity of a $\mathrm{Cu} / \mathrm{ZnO}$ Nanocatalyst via Gas Phase Pressure. Phys. Rev. Lett. 2013, 110 (8), 1-5.

(44) Kamachi, T.; Yoshizawa, K. Low-Mode Conformational Search Method with Semiempirical Quantum Mechanical Calculations: Application to Enantioselective Organocatalysis. J. Chem. Inf. Model. 2016, 56 (2), 347-353.

(45) Perdew, J. P.; Burke, K.; Ernzerhof, M. Generalized Gradient Approximation Made Simple. Phys. Rev. Lett. 1996, 77 (18), 3865.

(46) Grimme, S.; Ehrlich, S.; Goerigk, L. Effect of the Damping Function in Dispersion Corrected Density Functional Theory. J. Comput. Chem. 2011, 32 (7), 1456-1465.

(47) Grimme, S.; Antony, J.; Ehrlich, S.; Krieg, H. A Consistent and Accurate Ab Initio Parametrization of Density Functional Dispersion Correction (DFT-D) for the 94 Elements H-Pu. J. Chem. Phys. 2010, 132 (15), 154104.

(48) Vandevondele, J.; Krack, M.; Mohamed, F.; Parrinello, M.; Chassaing, T.; Hutter, J. Quickstep: Fast and Accurate Density Functional Calculations Using a Mixed Gaussian and Plane Waves Approach. Comput. Phys. Commun. 2005, 167 (2), 103-128. 
(49) Labute, P. LowModeMD-Implicit Low-Mode Velocity Filtering Applied to Conformational Search of Macrocycles and Protein Loops. J. Chem. Inf. Model. 2010, 50 (5), 792-800.

(50) Shao, J.; Tanner, S. W.; Thompson, N.; Cheatham, T. E. Clustering Molecular Dynamics Trajectories: 1. Characterizing the Performance of Different Clustering Algorithms. J. Chem. Theory Comput. 2007, 3 (6), 2312-2334.

(51) De Paris, R.; Quevedo, C. V.; Ruiz, D. D.; Norberto de Souza, O.; Barros, R. C. Clustering Molecular Dynamics Trajectories for Optimizing Docking Experiments. Comput. Intell. Neurosci. 2015, $2015,916240$.

(52) Jin, X.; Han, J. K-Medoids Clustering. In Encyclopedia of Machine Learning; Sammut, C., Webb, G. I., Eds.; Springer US: Boston, MA, 2010; pp 564-565, DOI: 10.1007/978-0-387-301648426.

(53) Van Der Maaten, L.; Hinton, G. Visualizing Data Using T-SNE. J. Mach. Learn. Res. 2008, 9, 2579-2625.

(54) Davis, R. A.; Lii, K.-S.; Politis, D. N. Remarks on Some Nonparametric Estimates of a Density Function. In Selected Works of Murray Rosenblatt; Springer, 2011; pp 95-100.

(55) Simoudis, E.; Han, J.; Fayyad, U. Proceedings of the Second International Conference on Knowledge Discovery \& Data Mining; AAAI Press: Menlo Park, CA (United States), 1996. 